\title{
Employing Feed-Forward Downstream Cancellation in Optical Network Units for 2.5G/1.25G RSOA-based and 10G/10G REAM- based Passive Optical Networks for Efficient Wavelength Reuse
}

\author{
Bernhard Schrenk, Jose A. Lazaro, Member, IEEE, Josep Prat, Member, IEEE \\ Universitat Politécnica de Catalunya, Jordi Girona 1-3, 08034 Barcelona, Spain \\ Tel: (3493) 401 7179, Fax: (3493) 401 7200,e-mail: bernhard.schrenk@tsc.upc.edu
}

\begin{abstract}
Optical Network Units for Next-Generation Passive Optical Networks with wavelength reuse are investigated for asymmetrical $2.5 \mathrm{G} / 1.25 \mathrm{G}$ and symmetrical $10 \mathrm{G} / 10 \mathrm{G}$ down- and upstream data rates. The transmission performance was enhanced by a feed-forward cancellation circuitry adapted to an RSOA and an REAM used as remodulator. The range of downstream extinction ratios for error-free upstream transmission at BER of $10^{-10}$ was extended from 3 to $4 \mathrm{~dB}$ for the RSOA-based ONU and from 1.5 to $3 \mathrm{~dB}$ for the REAM-based ONU.
\end{abstract}

Keywords: passive optical networks, WDM, RSOA, REAM, downstream cancellation, wavelength reuse.

\section{INTRODUCTION}

Fibre-to-the-Home access networks have been proven for delivering services with high bandwidth demands. Passive Optical Networks (PON) with a signal distribution assisted by Wavelength Division Multiplexing (WDM) seem to be good candidates [1]. However, there are several requirements for cost-effectiveness, such as scalability, robustness and a completely passive distribution network without active components. Several light sources and key components are then located at the Optical Line Terminal (OLT). High data rate and a high number of customers are further demands. To minimize its cost, the design of the Optical Network Unit (ONU) is a critical issue due to its desired mass deployment. A reflective, colourless ONU, e.g. based on a Reflective Semiconductor Optical Amplifier (RSOA), without wavelength-specific components is a beneficial solution [2].

Furthermore, means of wavelength reuse ensure that the capacity of the PON can be kept high. Although orthogonal modulation formats would be a good approach, they suffer from increased complexity at the costsensitive ONU: while phase modulation requires a photonic integrated interferometric demodulator to stay insensitive to temperature, frequency modulation is prohibited due to the specific filter needed for demodulation.

The lowest cost asks for the reuse of a single wavelength by keeping the simple intensity modulation format for both, down- and upstream. This faces the challenge of finding an optimum extinction ratio (ER) of the downstream to keep some residual power during its space bits for remodulation with upstream data: too low downstream ERs result in an unacceptable high penalty for its detection [3], while a too high ER degrades the upstream performance. Different schemes have been proposed to enhance the remodulation quality by means of downstream cancellation [4]-[7]. The downstream pattern is thereby erased by optical and electrical means to recover an optical carrier. These schemes differ in the requirements as well as in their remodulation quality.

\section{DOWNSTREAM CANCELLATION TECHNIQUES}

One approach to fill up the spaces in a present bit stream is to take advantage of the relative low optical gain saturation in a SOA [4]-[5]. This downstream compression is caused by the higher gain for the spaces over the marks. Although no electrical components are necessary, this method obviously suffers from relative high input power levels that are necessary to reach the saturation regime of the SOA, not always available in PONs.

Another technique is based on signal manipulation in the electrical domain. The detected signal is fed forward to the remodulating device, which is assumed to be an intensity modulator by its nature, to counteract the pattern. The level of the marks is brought to the lower one of the spaces, and a flat optical carrier suitable to embed the upstream is recreated. First results [6]-[7] have been shown at low data rates.

Despite techniques of cancellation, the residual downstream with ER $\varepsilon_{d s, r e s}$ at the OLT receiver will worsen the upstream transmission. Due to the electrical lowpass filter at the receiver that is adjusted to the upstream bit rate $B$, PONs with asymmetrical data rates for down- and upstream will be less vulnerable as the remaining downstream (amplitude $A_{d s, r e s}$ ) with the typically higher data rate will be cut in its spectrum $\Xi(f)$. Treating the downstream as Gaussian noise, which is an acceptable approximation [8], the $Q$-parameter for the upstream is

$$
Q=\frac{I_{1}-I_{0}}{\sigma_{0}+\sqrt{\sigma_{1}^{2}+\int_{-B_{e l}}^{B_{e l}} \Xi(f) d f}} \quad \Xi(f) \propto A_{d s, r e s}=2 I_{1} \frac{\varepsilon_{d s, r e s}-1}{\varepsilon_{d s, r e s}+1}
$$

where $I_{1}$ and $I_{0}$ are the average photo currents after detection for marks and a spaces, and $\sigma_{1}$ and $\sigma_{0}$ are their variances due to thermal, shot and beat noises from signal-ASE and ASE-ASE. It is assumed that the ER of the 
remodulator at the ONU is sufficient high so that a residual downstream at the space level is negligible low and no additional contribution is suffered from. For a symmetrical data rate, there will be an unsuppressed noise contribution, and it is the cancellation technique itself that is responsible for an enhanced upstream transmission.

In this work, the focus is put on a feed-forward cancellation for a non restricted operating point of the ONU to high optical input power levels. Furthermore, electrical filtering consisting of equalisation for the electrooptical (e/o) interface of the remodulator, to enhance its bandwidth limitations at the cost of some RF losses, and also derivative filtering in the feed-forward path, to give emphasis to the high bit rate transients, is taken into account. This is crucial for asymmetrical data rates when the modulating device for the upstream is drawn for the upstream with a lower data rate. The cancellation current $i_{f f}$ is composed out of the detected downstream $i_{D S}$ by

$$
i_{f f}(t+\Delta \tau)=-G_{f f}\left(i_{D S}(t)+k \frac{\partial i_{D S}(t)}{\partial t}\right) \Leftrightarrow \quad I_{f f}(j \omega)=-G_{f f} I_{D S}(j \omega)(1+j \omega k) e^{j \omega \Delta \tau}
$$

where the gain $G_{f f}$ of the feed-forward path and its delay $\Delta \tau$ are adjusted to the power level and ER of the downstream and the electrical and optical path length. The magnitude $k$ of the derivative is chosen according to the gain relaxation time of the RSOA which was 170 ps. In this way, its overshoot caused by the ER in the downstream is not further pronounced once the cutoff frequency of the derivative filter is kept low enough.

The ONU design chosen for the feed-forward cancellation depends on the bit rate to keep simplicity: it is considered an RSOA for low bit rate gain modulation and an REAM for faster modulation (Fig. 1).

The RSOA is suitable for a PON that features a $2.5 \mathrm{~Gb} / \mathrm{s}$ downstream with a Pseudo-Random Bit Sequence (PRBS) of length $2^{23}-1$, that is remodulated with $1.25 \mathrm{~Gb} / \mathrm{s}$ upstream data having a PRBS of length $2^{31}-1$ (Fig. 1 , left ONU). A 50/50 coupler splits detection and remodulation branch, whereby the latter holds a fibre delay line (FDL) to cope for the slower propagation of the electronical cancellation signal that originates at the APD. The relation between gain and bias current requires an inverted data signal for the cancellation. The cancellation circuit itself consists of an electrical delay $(\Delta \tau)$ for fine tuning of the path lengths, a derivative filter (DF) as described before, and RF amplifiers. A passive combiner was taken to add the upstream to the cancellation signal. The Equaliser (EQ) that enhances the e/o response from 0.8 to $1.2 \mathrm{GHz}$ was put in front of the RSOA.

For a higher symmetrical data rate of $10 \mathrm{~Gb} / \mathrm{s}$ and a PRBS length of $2^{7}-1$, equivalent to the requirements in Ethernet PON systems, an REAM replaces the RSOA as it offers a higher e/o bandwidth (Fig. 1, right ONU). The intrinsic losses of $16 \mathrm{~dB}$ restrict to use this device solely as the ONU would then provide a too low output power to cover a typical power budget of a PON. The missing gain is provided by a SOA at the ONU input which not only compensates for the losses, but also acts as preamplifier for the downstream. Due to the high e/o bandwidth $(12 \mathrm{GHz})$ provided by the REAM, the cancellation circuit does not require specific electrical filtering.

The remodulation quality will be proven in terms of carrier recovery and bit error ratio (BER) measurements. The experimental setup is shown in Fig. 1. The OLT transmitter consists of a Mach-Zehnder modulator (MZM) and an Erbium-doped fibre amplifier (EDFA) used as booster $\left(\mathrm{OA}_{\mathrm{B}}\right)$. The downstream ER was varied to proof the cancellation. The wavelength was $1551.32 \mathrm{~nm}$ for the RSOA-based ONU and $1560.61 \mathrm{~nm}$ for the REAMbased ONU. The launched power at the OLT was $3 \mathrm{dBm}$. For the BER measurements, the ONU and OLT input levels were varied with attenuators $\left(A_{F}, A_{R}\right)$, while the ONU input was fixed to $-14 \mathrm{dBm}$ for the upstream measurements. A combination of EDFA $\left(\mathrm{OA}_{\mathrm{P}}\right)$ and PIN diode was chosen as optical OLT receiver. A bandpass filter with a bandwidth of $60 \mathrm{GHz}$ was used to reject the ASE. The residual polarization sensitivity requires controllers. This would not be needed for future devices that are supposed to be insensitive.

The RSOA for the low bit rate ONU, having a noise figure of $9 \mathrm{~dB}$ and a small signal gain of $21.7 \mathrm{~dB}$ with an optical $3 \mathrm{~dB}$ gain bandwidth of $54 \mathrm{~nm}$ centred at $1550 \mathrm{~nm}$, was biased at a current of $55 \mathrm{~mA}$ and modulated with $70 \mathrm{~mA}_{\mathrm{pp}}$. The achieved upstream ER was $8 \mathrm{~dB}$ and is limited by the requirement of keeping the RSOA transparent during the space bits. For the high bit rate ONU, the SOA at the input, which had a small signal gain of $21 \mathrm{~dB}$ at its amplified spontaneous emission (ASE) peak, was biased with $70 \mathrm{~mA}$. The bias of the REAM was $-1.8 \mathrm{~V}$, while the modulation was $3.5 \mathrm{~V}_{\mathrm{pp}}$. An ER of $13 \mathrm{~dB}$ was obtained for the upstream. No cross-gain modulation was observed in the common SOA, which is attributed to the high intrinsic losses of the REAM.
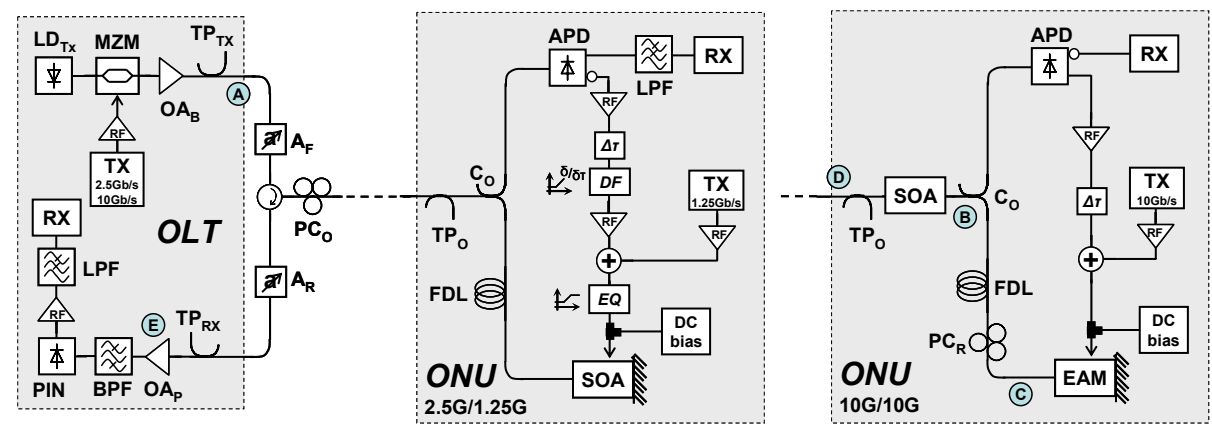

Figure 1. Setup for proving feed-forward downstream cancellation on a RSOA-based and a REAM-based ONU. 

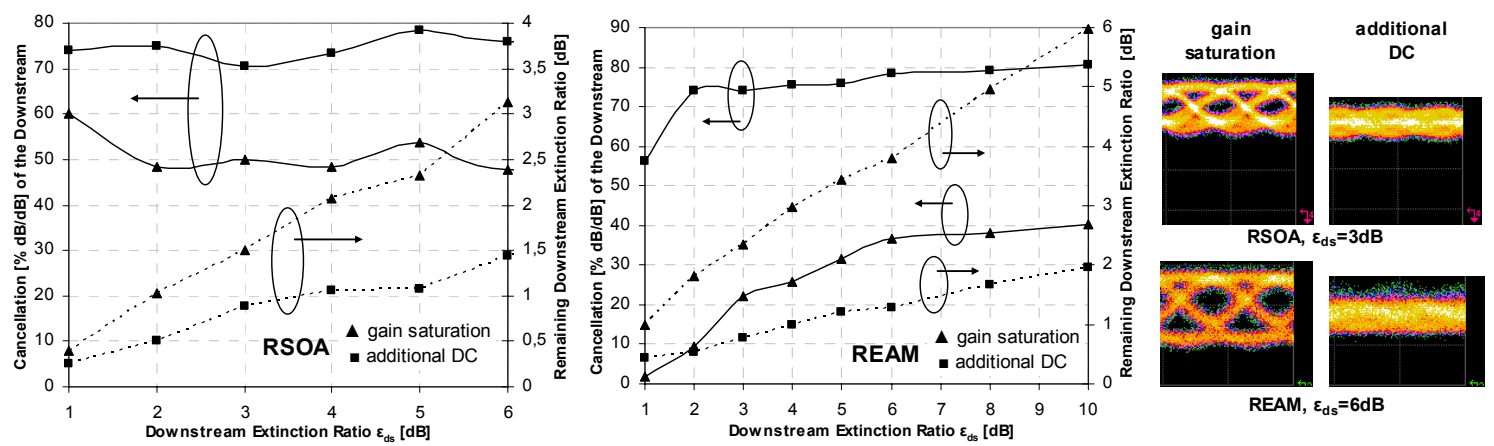

Figure 2. Cancellation efficiency and remaining downstream for carrier recovery using RSOA and REAM.

For an input power of $-14 \mathrm{dBm}$ at this ONU, the power levels and Optical Signal-to-Noise Ratios (OSNR) are: $3 \mathrm{dBm}$ and $46 \mathrm{~dB}$ after the OLT booster (point A in Fig. 1), $-1.5 \mathrm{dBm}$ and $38.8 \mathrm{~dB}$ after amplification with the SOA (B), $-20.5 \mathrm{dBm}$ after remodulation with the REAM (C), and $-12.1 \mathrm{dBm}$ and $27.7 \mathrm{~dB}$ after being boosted by the SOA (D). The ONU had a net gain of $1.9 \mathrm{~dB}$. The noise figure of the OLT preamplifier (E) was $4 \mathrm{~dB}$.

\section{RESULTS AND DISCUSSION}

The efficiency of natural optical gain saturation and additional feed-forward cancellation (DC) to counteract a downstream is presented in Fig. 2, for an input power into the ONU of $-14 \mathrm{dBm}$. With the RSOA as remodulator, an electrical filter with a bandwidth of $1.25 \mathrm{GHz}$ was placed after the upstream detector, while there was no upstream modulation itself. The efficiency increases by $21 \%$ for an ER of $3 \mathrm{~dB}$, where the remaining ER is $1.5 \mathrm{~dB}$ with the gain saturation effect and $0.9 \mathrm{~dB}$ with additional feed-forward cancellation. For the REAMbased ONU, the efficiency for an ER of $6 \mathrm{~dB}$ increases by $42 \%$ employing electrical means of cancellation, where the remaining ER decreases from $3.8 \mathrm{~dB}$ to $1.3 \mathrm{~dB}$. For both ONUs, the efficiency is constant for high downstream ERs, while for lower ones distortions due to the overshoot of the SOA reduce the efficiency.

The downstream BER measurements for the RSOA-based ONU are shown in Fig. 3. Considering a BER of $10^{-10}$, ERs bigger than $2 \mathrm{~dB}$ are reasonable for a typical power budget of a PON. The sensitivity for an ER of $3 \mathrm{~dB}$ is $-18.7 \mathrm{dBm}$. For an ER of $1 \mathrm{~dB}$, an error floor appears above a BER of $10^{-10}$. By contributing Forward Error Correction (FEC) with a BER threshold of $10^{-4}$, an ER of $2 \mathrm{~dB}$ with a sensitivity of $-18.4 \mathrm{dBm}$ would be suitable. The penalty for an ER of $3 \mathrm{~dB}$ is $8.5 \mathrm{~dB}$ for a BER of $10^{-10}$, referenced to an ER $>10 \mathrm{~dB}$. For the upstream (Fig. 4), a BER of $10^{-10}$ was obtained for ERs of $3 \mathrm{~dB}$ and less without, and also for $4 \mathrm{~dB}$, with cancellation. The sensitivity is then $-28.8 \mathrm{dBm}$ and the penalty referenced to the case without downstream is $7 \mathrm{~dB}$. At an ER of $3 \mathrm{~dB}$, the decrease of penalty given by the cancellation circuit is $4.2 \mathrm{~dB}$. The FEC threshold can be reached even with an ER of $6 \mathrm{~dB}$. For this ER, the sensitivity increases by $4.7 \mathrm{~dB}$ once feed-forward cancellation is used.
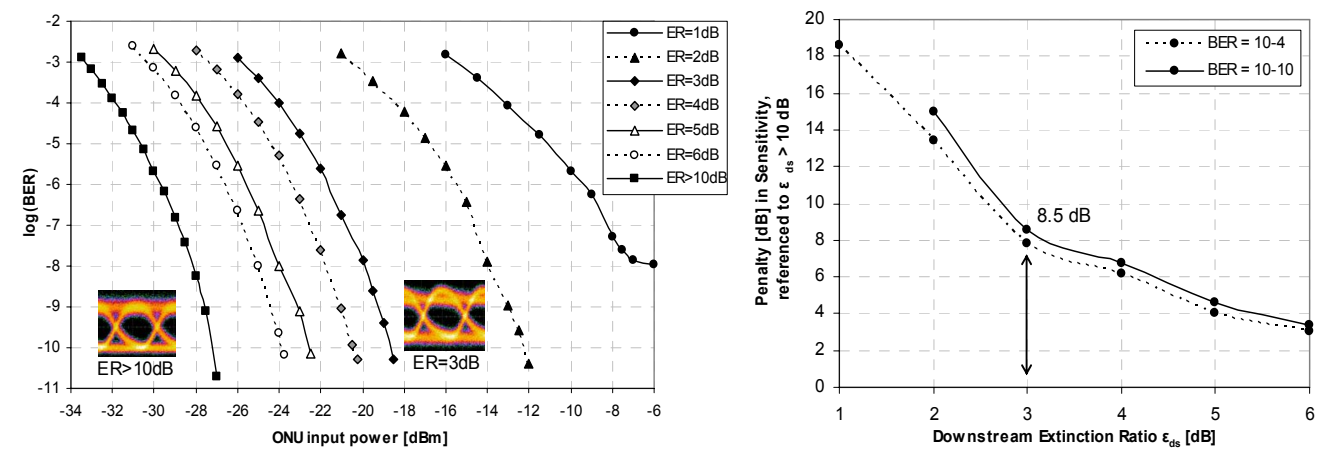

Figure 3. Downstream BER measurements for RSOA-based ONU and its penalty in sensitivity.
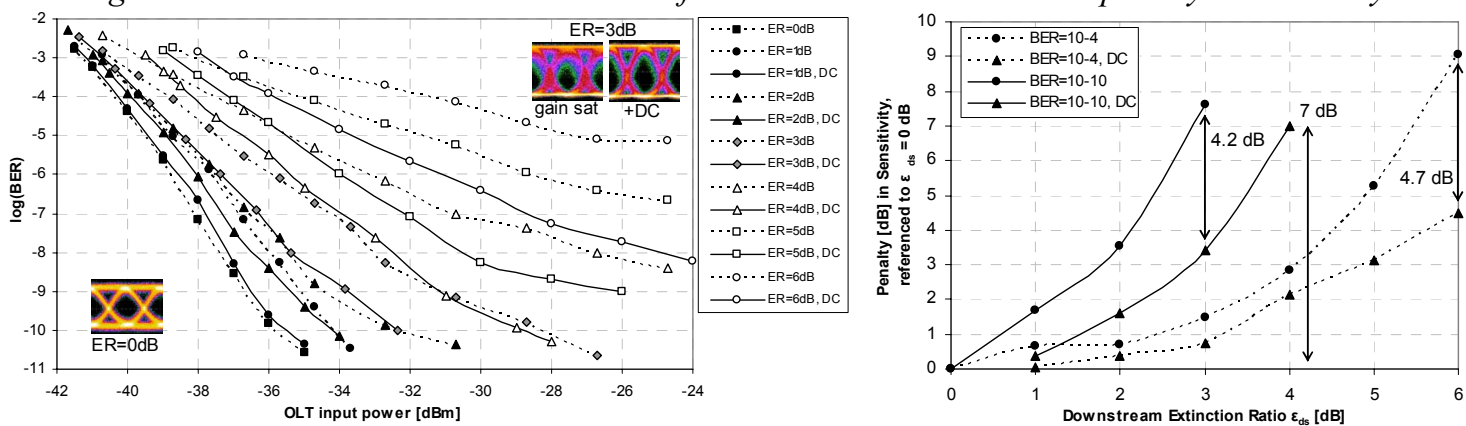

Figure 4. Upstream BER measurements for RSOA-based ONU and its penalty in sensitivity. 

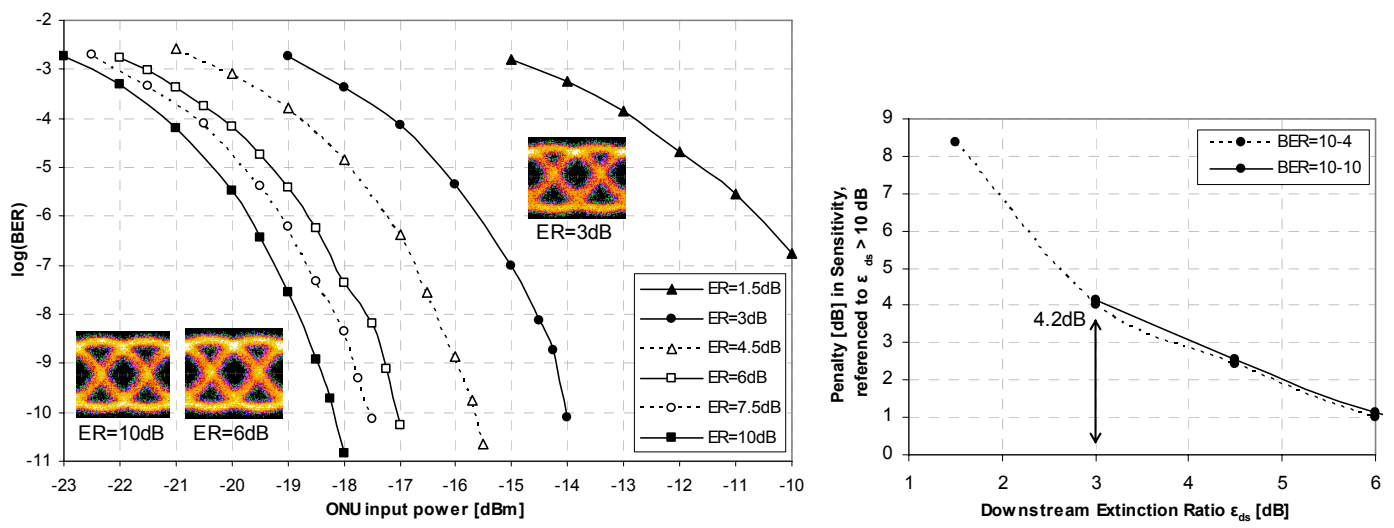

Figure 5. Downstream BER measurements for REAM-based ONU and its penalty in sensitivity.
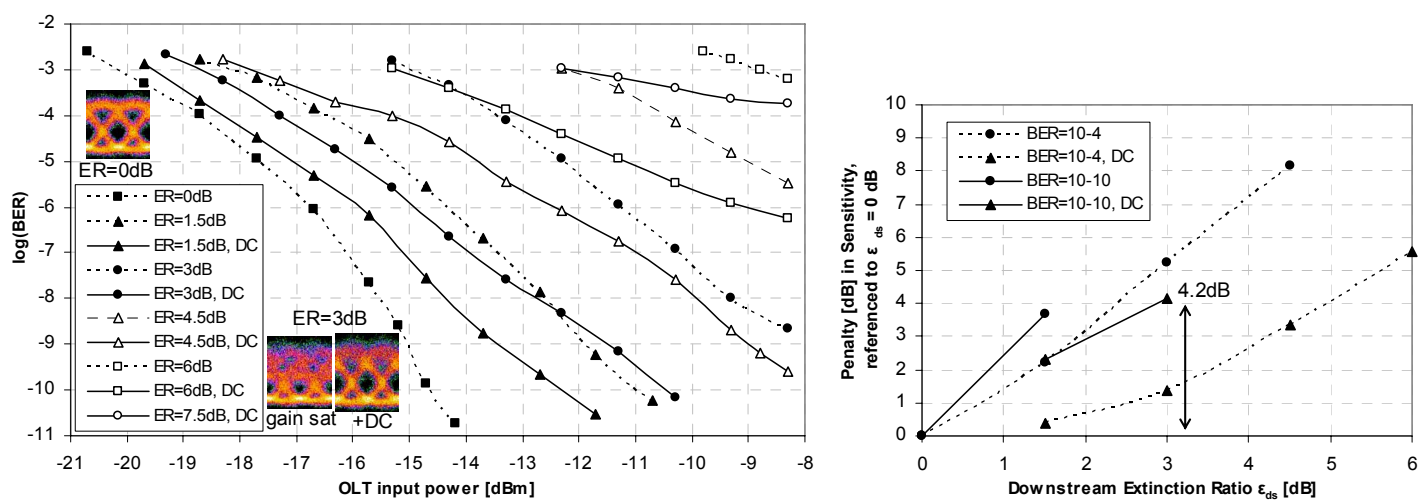

Figure 6. Upstream BER measurements for REAM-based ONU and its penalty in sensitivity.

For the REAM-based ONU, reasonable ERs for the downstream (Fig. 5) start with $3 \mathrm{~dB}$, having a sensitivity of $-14 \mathrm{dBm}$ at a BER of $10^{-10}$, where the penalty is $4.2 \mathrm{~dB}$. Operation at the FEC threshold does not give a crucial advantage. For the upstream (Fig. 6), the operating range for a BER of $10^{-10}$ can be extended from an ER of $1.5 \mathrm{~dB}$ to $3 \mathrm{~dB}$ when employing the cancellation. The sensitivity is then just $-10.5 \mathrm{dBm}$ due to the low net gain of the ONU, giving a penalty of $4.2 \mathrm{~dB}$. At the FEC level, the ER can be extended from $4.5 \mathrm{~dB}$ to at least $6 \mathrm{~dB}$.

\section{CONCLUSIONS}

The downstream ER was expanded by means of feed-forward cancellation in the ONU. It was passed from a non compatible 10G/10G down- and upstream situation to a full-duplex configuration that provides a BER of $10^{-10}$ for an ER of $3 \mathrm{~dB}$. The upstream was found to be critical due to the smaller margin towards the power budget.

\section{ACKNOWLEDGEMENTS}

This work was supported by the European FP7 Sardana and the Spanish MICINN TEC2008-01887 project.

\section{REFERENCES}

[1] A. Banerjee et al:: Wavelength-division-multiplexed passive optical network (WDM-PON) technologies for broadband access: a review, J. Optical Networking, vol. 4. pp. 737-758, Nov. 2005.

[2] J. Prat et al.: Optical network unit based on a bidirectional reflective semiconductor optical amplifier for fiber-to-the-home networks, Phot. Tech. Lett., vol. 17, pp. 250-252, Jan. 2005

[3] J.J. Koponen, M.J. Söderlund: A duplex WDM passive optical network with 1:16 power split using reflective SOA remodulator at ONU, in Proc. OFC 2004, Los Angeles, U.S., Feb. 2004, paper MF99.

[4] H. Takesue, T. Sugie: Wavelength channel data rewriter using semiconductor optical saturator/modulator, J. Lightwave Tech., vol. 24, pp. 2347-2354, June 2006.

[5] J.H. Yu, N. Kim, B.W. Kim: Remodulation schemes with reflective SOA for colorless DWDM PON, J. Optical Networking, vol. 6, pp. 1041-1054, Aug. 2007.

[6] W. Lee et. al.: Optical transceiver employing an RSOA with feed-forward current injection," in Proc. OFC 2007, Anaheim, U.S., March 2007, paper OTuH1.

[7] E. Conforti et. al:: Carrier reuse with gain compression and feed-forward semiconductor optical amplifiers, Transactions on Microwave Theory and Techniques, vol. 50, pp. 77-81, Jan. 2002.

[8] S.D. Personick et. al: : A detailed comparison of four approaches to the calculation of the sensitivity of optical fiber system receivers, Transactions on Communication, vol. COM-25, pp. 541-548, May 1977. 